\title{
Association of Long-Term Exposure to Fine Particulate Matter and Cardio-Metabolic Diseases in Low- and Middle-Income Countries: A Systematic Review
}

\author{
Suganthi Jaganathan ${ }^{1, *}$, Lindsay M. Jaacks ${ }^{2}\left(\mathbb{D}\right.$, Melina Magsumbol ${ }^{3}{ }^{(D}$, Gagandeep K. Walia $^{3}$, \\ Nancy L. Sieber ${ }^{4}{ }^{\circ}$, Roopa Shivasankar ${ }^{1}$, Preet K. Dhillon ${ }^{3}$, Safraj Shahul Hameed ${ }^{1}$, \\ Joel Schwartz ${ }^{4}$ and Dorairaj Prabhakaran ${ }^{1,3,5}$ \\ 1 Centre for Chronic Disease Control, New Delhi 110016, India \\ 2 Department of Global Health and Population, Harvard T.H. Chan School of Public Health, Boston, \\ MA 02115, USA \\ 3 Public Health Foundation of India, Gurgaon 122002, India \\ 4 Department of Environmental Health, Harvard T.H. Chan School of Public Health, Boston, MA 02115, USA \\ 5 London School of Hygiene and Tropical Medicine, London WC1E 7HT, UK \\ * Correspondence: suganthi@ccdcindia.org; Tel.: +91-11-416-1 9456
}

Received: 17 May 2019; Accepted: 6 June 2019; Published: 16 July 2019

check for updates

\begin{abstract}
Background: Numerous epidemiological studies indicated high levels of particulate matter less than2.5 $\mu \mathrm{m}$ diameter $\left(\mathrm{PM}_{2.5}\right)$ as a major cardiovascular risk factor. Most of the studies have been conducted in high-income countries (HICs), where average levels of $\mathrm{PM}_{2.5}$ are far less compared to low- and middle- income countries (LMICs), and their socio-economic profile, disease burden, and PM speciation/composition are very different. We systematically reviewed the association of long-term exposure to $\mathrm{PM}_{2.5}$ and cardio-metabolic diseases (CMDs) in LMICs. Methods: Multiple databases were searched for English articles with date limits until March 2018. We included studies investigating the association of long-term exposure to $\mathrm{PM}_{2.5}$ (defined as an annual average/average measure for 3 more days of $\mathrm{PM}_{2.5}$ exposure) and CMDs, such as hospital admissions, prevalence, and deaths due to CMDs, conducted in LMICs as defined by World Bank. We excluded studies which employed exposure proxy measures, studies among specific occupational groups, and specific episodes of air pollution. Results: A total of 5567 unique articles were identified, of which only 17 articles were included for final review, and these studies were from Brazil, Bulgaria, China, India, and Mexico. Outcome assessed were hypertension, type 2 diabetes mellitus and insulin resistance, and cardiovascular disease (CVD)-related emergency room visits/admissions, death, and mortality. Largely a positive association between exposure to $\mathrm{PM}_{2.5}$ and CMDs was found, and CVD mortality with effect estimates ranging from $0.24 \%$ to $6.11 \%$ increased per $10 \mu \mathrm{g} / \mathrm{m}^{3}$ in $\mathrm{PM}_{2.5}$. CVD-related hospitalizations and emergency room visits increased by $0.3 \%$ to $19.6 \%$. Risk factors like hypertension had an odds ratio of 1.14, and type 2 diabetes mellitus had an odds ratio ranging from 1.14-1.32. Diversity of exposure assessment and health outcomes limited the ability to perform a meta-analysis. Conclusion: Limited evidence on the association of long-term exposure to $\mathrm{PM}_{2.5}$ and CMDs in the LMICs context warrants cohort studies to establish the association.
\end{abstract}

Keywords: air pollution; cardio-metabolic diseases

\section{Background}

Cardiovascular disease (CVD) is the leading cause of death in nearly all countries around the globe [1], and chronic exposure to air pollution is an important risk factor for CVD. Based on numerous epidemiological studies, particulate matter with a diameter of less than 2.5 micrometers $\left(\mathrm{PM}_{2.5}\right)$ 
is considered to be the main culprit for these adverse cardiovascular effects [2-4]. Evidence from systematic reviews and meta-analyses suggests a strong association between short-term exposure to elevated $\mathrm{PM}_{2.5}$ and hospital admissions, myocardial infarction, stroke, and heart failure [5-8]. Even in countries where current international air quality standards are met (i.e., the World Health Organization (WHO) standards of $10 \mu \mathrm{g} / \mathrm{m}^{3}$ annual mean concentration or $25 \mu \mathrm{g} / \mathrm{m}^{3}$ 24-hour mean concentration) [2,9-12], small increases in $\mathrm{PM}_{2.5}$ are associated with increased risk of stroke [13].

More recent evidence suggests that chronic, i.e., long-term exposure to elevated $\mathrm{PM}_{2.5}$ also contributes substantially to cardio-metabolic diseases (CMDs) [4,14]. A meta-analysis from European and North American studies reported that the association between $\mathrm{PM}_{2.5}$ and type 2 diabetes mellitus (T2DM) increased the risk by $10 \%$ for every $10 \mu \mathrm{g} / \mathrm{m}^{3}$ increase in $\mathrm{PM}_{2.5}$ [15], which was similar to a meta-analysis conducted in 2014 that found an $11 \%$ increase in T2DM risk per $10 \mu \mathrm{g} / \mathrm{m}^{3}$ increase in $\mathrm{PM}_{2.5}$ among five identified studies [16]. Similarly, a systematic review conducted in 2015 identified five studies on long-term $\mathrm{PM}_{2.5}$ exposure and hypertension risk, and meta-analysis revealed a small albeit statistically non-significant effect summary odds ratio $(\mathrm{OR})=1.06(95 \%$ confidence interval $(\mathrm{CI})$, $0.98,1.15)$ [17]. All of these reviews included only developed countries (US, Canada, and Europe).

Thus, while an association between $\mathrm{PM}_{2.5}$ and CMD has been demonstrated by epidemiological studies conducted in developed countries, where the annual average $\mathrm{PM}_{2.5}$ concentrations are near the WHO standard, scientific evidence from low- and middle-income countries (LMICs) is extremely limited [18]. This is concerning given substantially higher exposure levels in LMICs due to rapid industrialization and inadequate enforcement of environmental regulations [19]. Moreover, differences in the sources and composition of ambient air pollution, diet, and chronic disease status limit the generalizability of findings from developed countries [20]. To further define the current state of the science in LMICs, we conducted a systematic literature review of the association between long-term exposure to $\mathrm{PM}_{2.5}$ and CMDs in LMICs.

\section{Objective}

To systematically compile evidence for the association between long-term exposure to $\mathrm{PM}_{2.5}$ and CMDs in LMICs.

\section{Materials and Methods}

We performed this study in three stages: database search, title and abstract screening, and full-text review and extraction. First, we searched the following databases: Medline and Embase (from January 1, 1948 to March 6, 2018), Cumulative Index to Nursing and Allied Health Literature, SCOPUS, Cochrane database, and Web of Science. We restricted our search to articles published in English, and we only considered peer-reviewed original articles. Detailed search terms are provided in Appendix A.

\section{Definitions}

For the purpose of this review, we defined CMDs as a clustering of disorders (hypertension, lipid disorders, hyperinsulinemia, and glucose intolerance) that together lead to CVD and T2DM [21,22]. We focused specifically on long-term exposure to $\mathrm{PM}_{2.5}$, defined as an annual average or average measure of more than 3 days of $\mathrm{PM}_{2.5}$ exposure. We chose to focus on long-term exposure, given that the diseases of interest (CMDs) are chronic diseases that develop over long periods of time.

\subsection{Inclusion and Exclusion Criteria}

We included studies that:

(1) Were original research published in peer-reviewed journals up to March 6, 2018.

(2) Were conducted in an LMIC according to current World Bank classification [23]. 
(3) Quantified the association between exposure to $\mathrm{PM}_{2.5}$ and at least one $\mathrm{CMD}$, including CVD, T2DM, lipid disorders, hypertension, hyperinsulinemia, and glucose intolerance, and related hospitalizations.

(4) Involved long-term exposure to $\mathrm{PM}_{2.5}$ (annual average or more than 3 days).

We excluded studies that:

(1) Were conducted only among the population aged less than 18 years.

(2) Used proxy measures to assess the exposure to $\mathrm{PM}_{2.5}$ (e.g., proximity to major roads).

(3) Focused on a specific population (e.g., patients with previous CVD events or T2DM).

\subsection{Selection of Studies}

The search strategy followed the PICO-population, intervention/exposure, comparator (there was no comparator in our review) and outcomes process [24]: population-general population from LMICs, intervention/exposure- long-term exposure to $\mathrm{PM}_{2.5}$, and outcomes - CMDs. Titles and abstracts were screened by three researchers (S.J., G.K.W., and M.M.). A full-text review was then performed independently by three researchers (S.J., G.K.W., and M.M). Disagreements were resolved by discussion in a small working group (S.J., G.K.W., M.M., and L.M.J.). Studies were then appraised for quality using the Cochrane Risk of Bias tool [25]. Each study was assessed for the following: selection bias, assessment of exposure and outcome, and adjustment for confounders. The studies were classified into three groups: high-quality (low risk of bias), medium-quality (unclear), and low-quality (high risk of bias). Two reviewers (S.J. and G.K.W.) independently appraised the studies, and disagreements were resolved in a consensus meeting. Only high-quality studies were included in this review.

\subsection{Data Extraction}

Data were extracted using a standardized extraction table that was piloted for two articles. Data extraction was performed by one researcher (S.J.) and was checked for accuracy by a small working group (G.K.W., M.M., and L.M.J.). The data extraction included study characteristics (author, year of publication, year of data collection, region, country, sampling method, sample size, location, setting), demographics (sex and age distribution), air pollution exposure information (method of measurement, length of exposure, and average measure), CMDs (prevalence/admissions/mortality, source of information), and results.

\section{Results}

\subsection{Study Selection Process}

We identified 10,861 articles in the database search, of which 5,291 were excluded as duplicates (Figure 1). Title and abstracts were screened, and 45 studies were identified for full-text review. Twenty-eight articles were excluded mainly because they were not original research $(n=8)$, did not assess $\mathrm{PM}_{2.5}(n=3)$, assessed short-term $\mathrm{PM}_{2.5}$ exposure $(n=11)$, did not measure an association $(n=1)$, used proxy measures of $\mathrm{PM}_{2.5}$ exposure $(n=1)$, or were in patients with a pre-existing condition $(n=4)$. The final review included 17 studies (Tables 1 and 2$)$. 

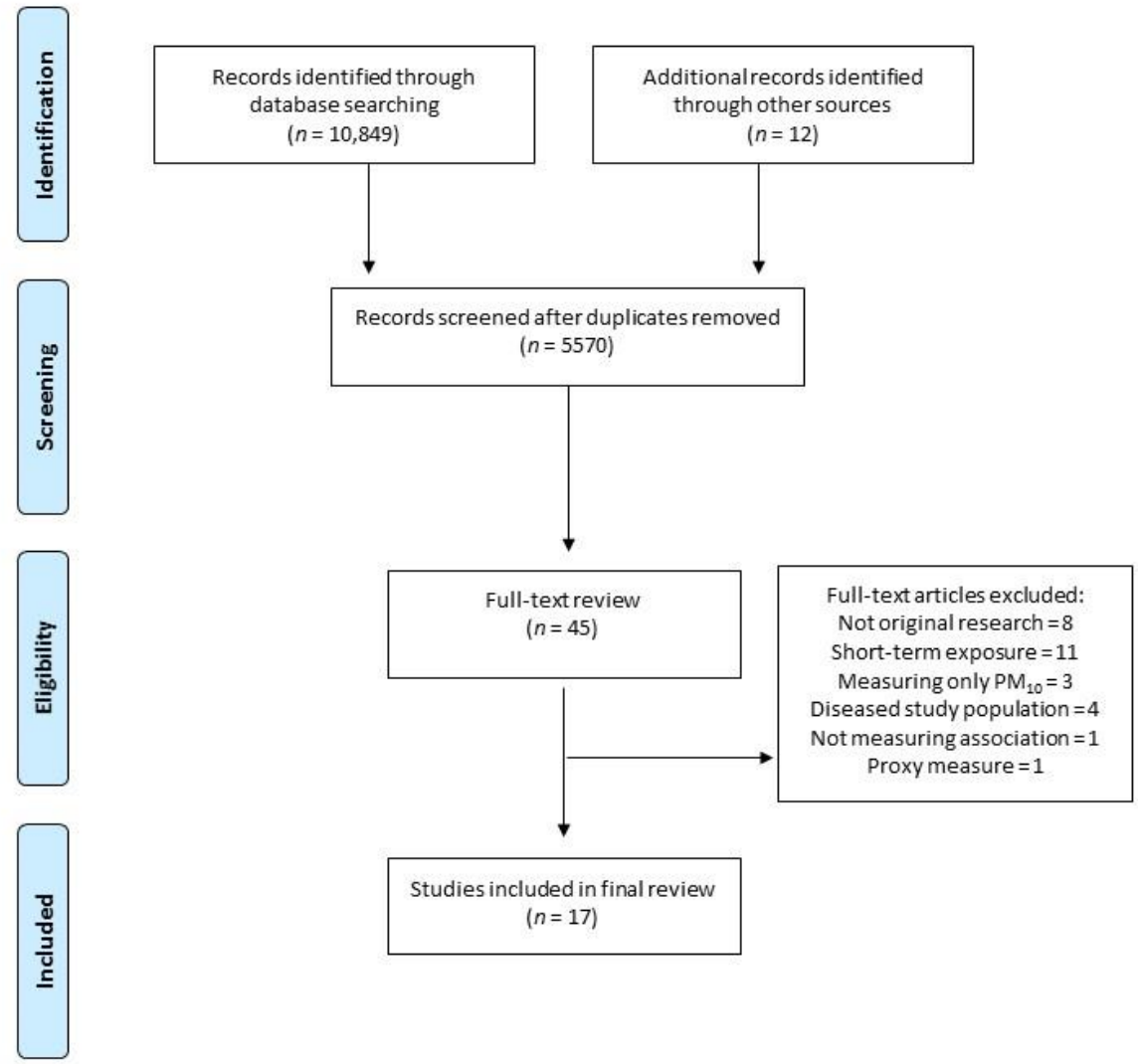

Figure 1. PRISMA flowchart [26]. 
Table 1. Study characteristics, exposure, outcome, and primary results of included studies ( $n=11$, listed alphabetically) measuring PM 2.5 exposure less than the annual average.

\begin{tabular}{|c|c|c|c|c|c|c|c|c|c|c|}
\hline Citation & $\begin{array}{l}\text { Place of } \\
\text { Study }\end{array}$ & $\begin{array}{l}\text { Study } \\
\text { Period }\end{array}$ & Study Design & $\begin{array}{l}\text { Study Population } \\
\text { (Age, Gender) }\end{array}$ & Sample Size & $\begin{array}{l}\text { Particulate Matter } \\
\text { Measuring Less Than } \\
2.5 \mu \mathrm{m}\left(\mathrm{PM}_{2.5}\right) \\
\text { Measurement } \\
\text { Method }\end{array}$ & $\begin{array}{l}\text { Mean } \\
\mathrm{PM}_{2.5} \\
\mu \mathrm{g} / \mathrm{m}^{3}\end{array}$ & $\begin{array}{l}\text { Exposure } \\
\text { Association to } \\
\text { Outcome } \\
\text { (No. of Days } \\
\text { Exposure Data- } \\
\text { Available) }\end{array}$ & $\begin{array}{l}\text { Outcomes } \\
\text { (Source) }\end{array}$ & Results \\
\hline [27] & Mexico City & 1993-1995 & $\begin{array}{l}\text { Time-series } \\
\text { study }\end{array}$ & $\begin{array}{l}\text { Residents aged } 65 \\
\text { years and above }\end{array}$ & 4129 & $\begin{array}{l}\text { Monitoring station with } \\
\text { 24-hour integrated } \\
\text { particle mass }\end{array}$ & 27 & 4 days (941 days) & $\begin{array}{l}\text { Daily mortality } \\
\text { (Electronic } \\
\text { records) }\end{array}$ & $\begin{array}{l}\text { Every } 10 \mu \mathrm{g} / \mathrm{m}^{3} \text { increase } \\
\text { in } \mathrm{PM}_{2.5} \text { was associated } \\
\text { with a } 3.4 \%(95 \% \\
\text { confidence interval (CI) } \\
0.67,6.18) \text { increase in } \\
\text { cardiovascular disease } \\
\text { (CVD) mortality }\end{array}$ \\
\hline [28] & $\begin{array}{l}\text { Plovdiv, } \\
\text { Bulgaria }\end{array}$ & 2014 & $\begin{array}{l}\text { Cross-sectional } \\
\text { survey }\end{array}$ & $\begin{array}{l}\text { General } \\
\text { population aged } \\
18 \text { years and } \\
\text { above }\end{array}$ & 513 & $\begin{array}{l}\text { Official municipality } \\
\text { source }\end{array}$ & 66.8 & $\begin{array}{l}\text { Not Available } \\
\text { (NA) (150 days) }\end{array}$ & $\begin{array}{l}\text { Type } 2 \text { diabetes } \\
\text { mellitus (T2DM) } \\
\text { prevalence } \\
\text { (survey) }\end{array}$ & $\begin{array}{l}\text { T2DM was positively } \\
\text { associated with } \text { PM }_{2.5} \text { : } \\
\text { Odds Ratio(OR) } 1.32(95 \% \\
\text { CI } 0.28,6.24) \text { comparing } \\
\text { top quartile }(25.0-66.8 \\
\left.\mu \mathrm{g} / \mathrm{m}^{3}\right) \text { to bottom quartile } \\
\left(0.0-25.0 \mu \mathrm{g} / \mathrm{m}^{3}\right)\end{array}$ \\
\hline [29] & $\begin{array}{l}\text { São José dos } \\
\text { Campos, } \\
\text { Brazil }\end{array}$ & 2010-2011 & $\begin{array}{l}\text { Time-series } \\
\text { study }\end{array}$ & $\begin{array}{l}\text { Daily hospital } \\
\text { admissions in } \\
\text { elderly people ( } 60 \\
\text { years and above) }\end{array}$ & 1765 & $\begin{array}{l}\text { Gent Stacked Filter-like } \\
\text { sampler; Companhia } \\
\text { Ambiental do Estado de } \\
\text { São Paulo-CETESB } \\
\text { monitoring station }\end{array}$ & 4.4 & 5 days (350 days) & $\begin{array}{l}\text { CVD hospital } \\
\text { admissions } \\
\text { (Health Services } \\
\text { Information } \\
\text { Database) }\end{array}$ & $\begin{array}{l}\text { Every } 10 \mu \mathrm{g} / \mathrm{m}^{3} \text { increase } \\
\text { in } \mathrm{PM}_{2.5} \text { increased the } \\
\text { risk of CVD } \\
\text { hospitalization by } 19.6 \% \\
(95 \% \text { CI } 6.4,34.6)\end{array}$ \\
\hline [30] & $\begin{array}{l}\text { Six cities of } \\
\text { the Pearl } \\
\text { River Delta } \\
\text { region, } \\
\text { China }\end{array}$ & 2013-2015 & $\begin{array}{l}\text { Cross-sectional } \\
\text { survey }\end{array}$ & Deaths registered & 316,305 & $\begin{array}{l}\text { Monitoring stations in } \\
\text { each of the six cities: } \\
\text { Guangzhou, Shenzhen, } \\
\text { Zhuhai, Dongguan, } \\
\text { Foshan, and Jiangmen }\end{array}$ & $\begin{array}{l}35.1 \text { to } \\
47.9\end{array}$ & 4 days (886 days) & $\begin{array}{l}\text { CVD Mortality } \\
\text { (Guangdong } \\
\text { Provincial Center } \\
\text { for Disease } \\
\text { Control and } \\
\text { Prevention) }\end{array}$ & $\begin{array}{l}\text { Every } 10 \mu \mathrm{g} / \mathrm{m}^{3} \text { increase } \\
\text { in } \mathrm{PM}_{2.5} \text { concentration } \\
\text { was associated with } \\
2.19 \%(95 \% \text { CI 1.80, } 2.59) \\
\text { increase in CVD mortality }\end{array}$ \\
\hline [31] & $\begin{array}{l}\text { Guangzhou, } \\
\text { China }\end{array}$ & 2007-2011 & $\begin{array}{l}\text { Time-series } \\
\text { study }\end{array}$ & $\begin{array}{l}\text { General } \\
\text { population }\end{array}$ & 33,721 & $\begin{array}{l}\text { Panyu Meteorological } \\
\text { Center, South China } \\
\text { Institute of } \\
\text { Environmental Sciences } \\
\text { \& GRIMM Aerosol } \\
\text { Spectrometer }\end{array}$ & 41.4 & 4 days (1079 days) & $\begin{array}{l}\text { CVD mortality } \\
\text { (Guangdong } \\
\text { Provincial Center } \\
\text { for Disease } \\
\text { Control and } \\
\text { Prevention) }\end{array}$ & $\begin{array}{l}\text { An inter-quartile range } \\
(\mathrm{IQR}) \text { increase in } \mathrm{PM}_{2.5} \\
\left(31.5 \mu \mathrm{g} / \mathrm{m}^{3}\right) \text { was } \\
\text { associated with excess } \\
\text { risk of CVD mortality by } \\
6.11 \%(95 \% \text { CI } 1.76,10.64)\end{array}$ \\
\hline
\end{tabular}


Table 1. Cont.

\begin{tabular}{|c|c|c|c|c|c|c|c|c|c|c|}
\hline Citation & $\begin{array}{l}\text { Place of } \\
\text { Study }\end{array}$ & $\begin{array}{l}\text { Study } \\
\text { Period }\end{array}$ & Study Design & $\begin{array}{l}\text { Study Population } \\
\text { (Age, Gender) }\end{array}$ & Sample Size & $\begin{array}{l}\text { Particulate Matter } \\
\text { Measuring Less Than } \\
2.5 \mu \mathrm{m}\left(\mathrm{PM}_{2.5}\right) \\
\text { Measurement } \\
\text { Method }\end{array}$ & $\begin{array}{l}\text { Mean } \\
\mathrm{PM}_{2.5} \\
\mu \mathrm{g} / \mathrm{m}^{3}\end{array}$ & $\begin{array}{l}\text { Exposure } \\
\text { Association to } \\
\text { Outcome } \\
\text { (No. of Days } \\
\text { Exposure Data- } \\
\text { Available) }\end{array}$ & $\begin{array}{l}\text { Outcomes } \\
\text { (Source) }\end{array}$ & Results \\
\hline [32] & $\begin{array}{l}\text { Beijing, } \\
\text { China }\end{array}$ & 2004-2006 & $\begin{array}{l}\text { Time-series } \\
\text { study }\end{array}$ & $\begin{array}{l}\text { Emergency room } \\
\text { visits (ERV) }\end{array}$ & 13,026 & $\begin{array}{l}\text { Twin Differential } \\
\text { Mobility Particle Sizer } \\
\text { and Aerodynamic } \\
\text { Particle Sizer }\end{array}$ & 109.8 & $\begin{array}{l}11 \text { days (1035 } \\
\text { days) }\end{array}$ & $\begin{array}{l}\text { CVD ERV } \\
\text { (Emergency } \\
\text { Department of } \\
\text { Hospital) }\end{array}$ & $\begin{array}{l}\text { An IQR increase in } \mathrm{PM}_{2.5} \\
\left(43.0 \mu \mathrm{g} / \mathrm{m}^{3}\right) \text { was } \\
\text { associated with } 0.3 \%(95 \% \\
\mathrm{CI}(-) 2.4,3.0) \text { and }(-) 0.1 \\
(95 \% \text { CI } 3.4,3.3) \text { total and } \\
\text { severe CVD ERV, } \\
\text { respectively }\end{array}$ \\
\hline [33] & $\begin{array}{l}\text { Beijing, } \\
\text { China }\end{array}$ & 2008-2011 & $\begin{array}{l}\text { Time-stratified } \\
\text { case-crossover } \\
\text { study }\end{array}$ & Death registered & 145,477 & U.S. embassy & 95.7 & $\begin{array}{l}40 \text { days (1046 } \\
\text { days) }\end{array}$ & $\begin{array}{l}\text { CVD/ } \\
\text { Cerebrovascular } \\
\text { disease } \\
\text { (CBD)/Ischemic } \\
\text { Heart Disease } \\
\text { (IHD) (Death } \\
\text { Registry of } \\
\text { Chinese Center for } \\
\text { Disease Control } \\
\text { and Prevention) } \\
\end{array}$ & $\begin{array}{l}\text { Every } 10 \mu \mathrm{g} / \mathrm{m}^{3} \text { increase } \\
\text { in PM } 2.5 \text { was associated } \\
\text { with an increased risk of } \\
\text { CVD mortality of } 0.24 \% \\
(95 \% \text { CI } 0.11,0.39), \text { CBD } \\
\text { mortality of } 0.23 \%(95 \% \\
\text { CI } 0.03,0.42), \text { and IHD } \\
\text { mortality of } 0.22 \%(95 \% \\
\text { CI } 0.12,0.54)\end{array}$ \\
\hline [34] & $\begin{array}{l}\text { São José do } \\
\text { Rio Preto, } \\
\text { Brazil }\end{array}$ & 2011-2012 & $\begin{array}{l}\text { Time-series } \\
\text { study }\end{array}$ & $\begin{array}{l}\text { Hospitalizations } \\
\text { registered }\end{array}$ & 4505 & $\begin{array}{l}\text { Coupled Chemistry } \\
\text { Aerosol-Tracer } \\
\text { Transport model to the } \\
\text { Brazilian } \\
\text { developments on the } \\
\text { Regional Atmospheric } \\
\text { Modeling System } \\
\text { (CCATT BRAMS } \\
\text { model) }\end{array}$ & 23.8 & 5 days (365 days) & $\begin{array}{l}\text { CVD } \\
\text { hospitalization } \\
\text { (Unified Health } \\
\text { System) }\end{array}$ & $\begin{array}{l}\text { Every } 10 \mu \mathrm{g} / \mathrm{m}^{3} \text { increase } \\
\text { in } \mathrm{PM}_{2.5} \text { was associated } \\
\text { with a } 15 \% \text { increased } \\
\text { relative risk (RR) for CVD } \\
\text { hospitalization with SE of } \\
0.007 \%\end{array}$ \\
\hline [35] & $\begin{array}{l}\text { Shenyang, } \\
\text { China }\end{array}$ & 2006-2008 & $\begin{array}{l}\text { Time-series } \\
\text { study }\end{array}$ & $\begin{array}{l}\text { General } \\
\text { population }\end{array}$ & 32 & $\begin{array}{l}\text { Continuous monitoring } \\
\text { system at Shenyang } \\
\text { Regional } \\
\text { Meteorological Center; } \\
\text { Ambient Dust Monitor } \\
365\end{array}$ & 95.9 & 3 days (730 days) & $\begin{array}{l}\text { Cause specific } \\
\text { mortality } \\
\text { (Liaoning } \\
\text { Provincial Center } \\
\text { for Disease } \\
\text { Control and } \\
\text { Prevention) }\end{array}$ & $\begin{array}{l}\text { Every } 10 \mu \mathrm{g} / \mathrm{m}^{3} \text { increase } \\
\text { in } \mathrm{PM}_{2.5} \text { was associated } \\
\text { with a } 0.42 \%(95 \% \text { CI } 0.10 \text {, } \\
0.73) \text { increase in CVD } \\
\text { mortality }\end{array}$ \\
\hline [36] & $\begin{array}{l}\text { Cuiabá and } \\
\text { Várzea } \\
\text { Grande, } \\
\text { State of } \\
\text { Mato Grosso, } \\
\text { Brazil }\end{array}$ & 2009-2011 & $\begin{array}{l}\text { Time-series } \\
\text { study }\end{array}$ & $\begin{array}{l}\text { General } \\
\text { population aged } \\
45 \text { years and } \\
\text { above }\end{array}$ & $\begin{array}{l}8610 \\
\text { hospitalizations } \\
\text { and } 3024 \\
\text { deaths }\end{array}$ & $\begin{array}{l}\text { Method developed and } \\
\text { validated for the } \\
\text { Brazilian Amazon and } \\
\text { Cerrado }\end{array}$ & 17.7 & 10 days (983 days) & $\begin{array}{l}\text { Daily mortality } \\
\text { and } \\
\text { hospitalization } \\
\text { (Mortality } \\
\text { Information } \\
\text { System and } \\
\text { Hospital } \\
\text { Information } \\
\text { System) }\end{array}$ & $\begin{array}{l}\text { Every } 10 \mu \mathrm{gg} / \mathrm{m}^{3} \text { increase } \\
\text { in PM }{ }_{2.5} \text { was associated } \\
\text { with a } 2.64 \%(95 \% \text { CI } 0.53, \\
4.06) \text { increase in risk of } \\
\text { CVD hospitalizations and } \\
3.57 \%(95 \% \text { CI } 0.82,6.38) \\
\text { increase in CVD mortality }\end{array}$ \\
\hline
\end{tabular}


Table 1. Cont.

\begin{tabular}{|c|c|c|c|c|c|c|c|c|c|c|}
\hline Citation & $\begin{array}{l}\text { Place of } \\
\text { Study }\end{array}$ & $\begin{array}{l}\text { Study } \\
\text { Period }\end{array}$ & Study Design & $\begin{array}{l}\text { Study Population } \\
\text { (Age, Gender) }\end{array}$ & Sample Size & $\begin{array}{l}\text { Particulate Matter } \\
\text { Measuring Less Than } \\
2.5 \mu \mathrm{m}\left(\mathrm{PM}_{2.5}\right) \\
\text { Measurement } \\
\text { Method }\end{array}$ & $\begin{array}{l}\text { Mean } \\
\mathrm{PM}_{2.5} \\
\mu \mathrm{g} / \mathrm{m}^{3}\end{array}$ & $\begin{array}{l}\text { Exposure } \\
\text { Association to } \\
\text { Outcome } \\
\text { (No. of Days } \\
\text { Exposure Data- } \\
\text { Available) }\end{array}$ & $\begin{array}{l}\text { Outcomes } \\
\text { (Source) }\end{array}$ & Results \\
\hline [37] & $\begin{array}{l}\text { Chongqing, } \\
\text { China }\end{array}$ & 1995 & $\begin{array}{l}\text { Cross-sectional } \\
\text { survey }\end{array}$ & Deaths registered & 47 & $\begin{array}{l}\text { 24-hour samples } \\
\text { collected from two } \\
\text { roadside sites from } \\
\text { representative areas of } \\
\text { differing principal } \\
\text { social activities }\end{array}$ & 147 & 5 days (213 days) & $\begin{array}{l}\text { Daily mortality } \\
\text { (Chongqing } \\
\text { Anti-Epidemic } \\
\text { Station) }\end{array}$ & $\begin{array}{l}\text { Every } 100 \mu \mathrm{\mu g} / \mathrm{m}^{3} \text { increase } \\
\text { in PM }{ }_{2.5} \text { was associated } \\
\text { with } 1.09 \%(95 \% \text { CI } \\
0.95,1.20) \text { increase in CVD } \\
\text { mortality }\end{array}$ \\
\hline
\end{tabular}

CI-confidence interval; CVD-cardiovascular disease; PM-Particulate matter; NA-not available; T2DM-type 2 diabetes mellitus; OR-odds ratio; IQR-inter-quartile range; IHD-ishcemic heart disease; CBD-cerebrovascular disease; RR-relative risk. 
Table 2. Study characteristics, exposure, outcome, and primary results of included studies $\left(n=6\right.$, listed alphabetically) measuring $\mathrm{PM}_{2.5}$ exposure as annual average or more.

\begin{tabular}{|c|c|c|c|c|c|c|c|c|c|c|}
\hline Citation & Place of Study & $\begin{array}{l}\text { Study } \\
\text { Period }\end{array}$ & Study Design & $\begin{array}{l}\text { Study } \\
\text { Population } \\
\text { (Age, Gender) }\end{array}$ & $\begin{array}{l}\text { Sample } \\
\text { Size }\end{array}$ & $\begin{array}{l}\mathrm{PM}_{2.5} \\
\text { Measurement } \\
\text { Method }\end{array}$ & $\begin{array}{l}\text { Mean } \\
\mathrm{PM}_{2.5} \\
\mu \mathrm{g} / \mathrm{m}^{3}\end{array}$ & $\begin{array}{l}\text { Exposure } \\
\text { Association to } \\
\text { Outcome } \\
\text { (No. of Days } \\
\text { Exposure } \\
\text { Data-Available) } \\
\end{array}$ & Outcomes (Source) & Results \\
\hline [38] & Varanasi, India & 2001-2015 & $\begin{array}{l}\text { Time-series } \\
\text { study }\end{array}$ & $\begin{array}{l}\text { General } \\
\text { population }\end{array}$ & 5700 & $\begin{array}{l}\text { MODIS onboard } \\
\text { NASA-EOS AQUA } \\
\text { and TERRA satellites }\end{array}$ & 136.9 & 13 years (13 years) & $\begin{array}{l}\text { Premature mortality } \\
\text { from ischemic heart } \\
\text { disease (IHD), } \\
\text { Stroke (Premature } \\
\text { deaths) }\end{array}$ & $\begin{array}{l}\text { The estimated premature } \\
\text { deaths per year from } \\
\text { ambient } \mathrm{PM}_{2.5} \\
\text { exposure in Varanasi: } \\
\text { IHD } 1600(95 \% \text { CI 600, } \\
\text { 2200), Stroke } 1000(95 \% \\
\text { CI 500, 1400) }\end{array}$ \\
\hline [39] & $\begin{array}{l}\text { Shanghai and seven } \\
\text { provinces of } \\
\text { Guangdong, Hubei, } \\
\text { Jilin, Shaanxi, } \\
\text { Shandong, Yunnan, } \\
\text { and Zhejiang, China }\end{array}$ & 2007-2010 & $\begin{array}{l}\text { Cross-sectional } \\
\text { survey }\end{array}$ & $\begin{array}{l}\text { General } \\
\text { population } \\
\text { aged } 50 \text { years } \\
\text { and above }\end{array}$ & 12,665 & $\begin{array}{l}\text { van Donkelaar and } \\
\text { co-workers to } \\
\text { estimate the outdoor } \\
\mathrm{PM}_{2.5} \text { concentrations }\end{array}$ & 33.7 & 3 years (3 years) & $\begin{array}{l}\text { Hypertension } \\
\text { (World Health } \\
\text { Organistion (WHO) } \\
\text { Study on global } \\
\text { aging and adult } \\
\text { health) }\end{array}$ & $\begin{array}{l}\text { Odds ratio (OR) for } \\
\text { hypertension: } 1.14(95 \% \\
\mathrm{CI} 1.07,1.22) \text { for every } 10 \\
\mu \mathrm{g} / \mathrm{m}^{3} \text { increase in } \mathrm{PM}_{2.5}\end{array}$ \\
\hline [40] & National, China & 2011-2012 & $\begin{array}{l}\text { Cross-sectional } \\
\text { survey }\end{array}$ & $\begin{array}{l}\text { General } \\
\text { population } \\
\text { aged } \geq 45 \\
\text { years }\end{array}$ & 11,847 & $\begin{array}{l}\text { Satellite-based spatial } \\
\text { statistical model }\end{array}$ & 72.6 & 1 year (303 days) & $\begin{array}{l}\text { Type } 2 \text { diabetes } \\
\text { mellitus (T2DM) } \\
\text { prevalence, blood } \\
\text { glucose, and } \\
\text { HemoglobinA1c } \\
\text { (HbA1c) (survey) }\end{array}$ & $\begin{array}{l}\text { An inter-quartile range } \\
(\mathrm{IQR}) \text { increase in } \mathrm{PM}_{2.5} \\
\left(41.1 \mu \mathrm{\mu g} / \mathrm{m}^{3}\right) \text { was } \\
\text { associated with increased } \\
\text { T2DM prevalence ratio( } \\
\text { PR) } 1.14(95 \% \text { CI } 1.08, \\
1.20) \text {, elevated levels of } \\
\text { fasting glucose by } 0.26 \\
\text { mmol/L }(95 \% \mathrm{CI} 0.19, \\
0.32), \text { and HbA1c by } \\
0.08 \%(95 \% \mathrm{CI} 0.06,0.10)\end{array}$ \\
\hline [41] & National, China & 2015 & $\begin{array}{l}\text { Cross-sectional } \\
\text { survey }\end{array}$ & $\begin{array}{l}\text { General } \\
\text { population } \\
\text { aged } 20-49 \\
\text { years }\end{array}$ & $10,843,140$ & $\begin{array}{l}\text { Hybrid geophysical } \\
\text { statistical } \\
\text { approach }\end{array}$ & 47.1 & 3 years ( 3 years) & $\begin{array}{l}\text { Tachycardia and } \\
\text { Resting heart rate } \\
\text { (Survey) }\end{array}$ & $\begin{array}{l}\text { OR for tachycardia: } 1.018 \\
(95 \% \text { CI } 1.017,1.020) \text { and } \\
\text { a } 0.076(95 \% \text { CI } 0.073 \text {, } \\
0.079) \mathrm{bpm} \text { elevation in } \\
\text { the resting heart rate for } \\
\text { every } 10 \mu \mathrm{gg} / \mathrm{m}^{3} \text { increase } \\
\text { in } \mathrm{PM}_{2.5}\end{array}$ \\
\hline
\end{tabular}


Table 2. Cont.

\begin{tabular}{|c|c|c|c|c|c|c|c|c|c|c|}
\hline Citation & Place of Study & $\begin{array}{l}\text { Study } \\
\text { Period }\end{array}$ & Study Design & $\begin{array}{l}\text { Study } \\
\text { Population } \\
\text { (Age, Gender) }\end{array}$ & $\begin{array}{l}\text { Sample } \\
\text { Size }\end{array}$ & $\begin{array}{l}\mathrm{PM}_{2.5} \\
\text { Measurement } \\
\text { Method }\end{array}$ & $\begin{array}{l}\text { Mean } \\
\mathrm{PM}_{2.5} \\
\mu \mathrm{g} / \mathrm{m}^{3}\end{array}$ & $\begin{array}{l}\text { Exposure } \\
\text { Association to } \\
\text { Outcome } \\
\text { (No. of Days } \\
\text { Exposure } \\
\text { Data-Available) }\end{array}$ & Outcomes (Source) & Results \\
\hline [42] & Liaoning, China & 2009 & $\begin{array}{l}\text { Cross-sectional } \\
\text { survey }\end{array}$ & $\begin{array}{l}\text { General } \\
\text { population } \\
\text { aged } 18-74 \\
\text { years }\end{array}$ & 15,477 & $\begin{array}{l}\text { Satellite-based spatial } \\
\text { statistical model }\end{array}$ & 82.0 & 3 years (3 years) & $\begin{array}{l}\text { Prevalence of } \\
\text { diabetes, fasting } \\
\text { glucose, 2-hour } \\
\text { glucose, and 2-hour } \\
\text { insulin (Survey) }\end{array}$ & $\begin{array}{l}\text { An IQR increase in } \mathrm{PM}_{2.5} \\
\left(26 \mu \mathrm{g} / \mathrm{m}^{3}\right) \text { was } \\
\text { significantly associated } \\
\text { with increased diabetes: } \\
\text { OR } 1.14(95 \% \text { CI } 1.03 \text {, } \\
1.25)\end{array}$ \\
\hline [43] & National, China & 2000-2005 & Cohort study & $\begin{array}{l}\text { Males } 40 \text { years } \\
\text { and above }\end{array}$ & 189,793 & $\begin{array}{l}\text { Combination of } \\
\text { satellite-derived and } \\
\text { chemical transport } \\
\text { model estimates } \\
\text { calibrated to surface } \\
\text { measurements }\end{array}$ & 43.7 & 6 years (6 years) & $\begin{array}{l}\text { CVD Mortality } \\
\text { (Survey) }\end{array}$ & $\begin{array}{l}\text { Hazard ratio (HR) for } \\
\text { CVD mortality was } 1.09 \\
(95 \% \text { CI 1.08, } 1.10 \text { ) for } \\
\text { every } 10 \mu \mathrm{\mu g} / \mathrm{m}^{3} \text { increase } \\
\text { in } \mathrm{PM}_{2.5}\end{array}$ \\
\hline
\end{tabular}

CBD-Cerebrovascular Disease; CI-Confidence Interval; CVD-Cardiovascular Diseases; DBP-Diastolic Blood Pressure; T2DM-Type 2 Diabetes Mellitus; ER-Excess Risk; ERV-Emergency Room Visits; HbA1c-HemoglobinA1c; HR-Hazard Ratio; IHD-Ischemic Heart Disease; IQR-Interquartile Range; OR-Odds Ratio; PM-Particulate Matter; PR-Prevalence ratio; RR-Relative Risk; SBP-Systolic Blood Pressure. 
The following study designs were used in the included studies: seven were cross-sectional studies, eight were time-series studies, one was a time-stratified case-crossover study, and one was a cohort study. The included studies were conducted in the following countries: Brazil $(n=3)$, Bulgaria $(n=1)$, China $(n=11)$, India $(n=1)$, and Mexico $(n=1)$. Only two studies were conducted before 2008, and the rest were conducted after 2008 (past 10 years) on the studied outcomes.

\subsection{Study Characteristics: Exposure and Outcomes}

The most common methods of exposure assessment used in these studies were modeled estimates from hybrid space-time models, which use a combination of satellite remote sensing, meteorology, and land use as predictors $(n=8)$; data from nearby monitoring stations $(n=4)$; both direct measurements of $\mathrm{PM}_{2.5}$ and data from monitoring stations $(n=3)$ and direct measurement using personal monitors $(n=2)$. We could not perform meta-analyses due to the heterogeneity of $\mathrm{PM}_{2.5}$ measurement methods across studies. The average concentration of $\mathrm{PM}_{2.5}$ reported in all of the Chinese studies was 73.85 $\mu \mathrm{g} / \mathrm{m}^{3}$ ranging from $33.7 \mu \mathrm{g} / \mathrm{m}^{3}$ to $147 \mu \mathrm{g} / \mathrm{m}^{3}$. $\mathrm{PM}_{2.5}$ concentrations reported by studies conducted in India (2015), Brazil (2010-2012), Bulgaria (2014), and Mexico (1995) were $136.9 \mu \mathrm{g} / \mathrm{m}^{3}, 4.4$ to $23.8 \mu \mathrm{g} / \mathrm{m}^{3}$, $66.8 \mu \mathrm{g} / \mathrm{m}^{3}$, and $27 \mu \mathrm{g} / \mathrm{m}^{3}$, respectively. With respect to CMD outcomes reported in the included studies, three studies evaluated hospital admissions and emergency room visits (ERV); nine studies evaluated CVD mortality; five studies evaluated CVD risk factors, including T2DM. Table 1 summarizes 11 studies which evaluated shorter $\mathrm{PM}_{2.5}$ exposure windows ( 3 days up to 40 days), and Table 2 summarizes six studies which evaluated longer $\mathrm{PM}_{2.5}$ exposure windows (annual average up to 13-year average). The exposure association to outcome grouped the included studies into two categories, namely studies measuring less than annual averages of exposure and studies measuring an annual average of exposure with an exact number of days of exposure assessment mentioned in the Tables 1 and 2.

\subsection{Mortality Outcomes}

Of the nine studies that evaluated CVD mortality [27,31,33,35-38,43,44], eight (91\%) reported significant effects of long-term $\mathrm{PM}_{2.5}$ exposure. Of these studies, six were conducted in China, and one study each was carried out in Mexico, India, and Brazil. For every $10 \mu \mathrm{g} / \mathrm{m}^{3}$ increase in $\mathrm{PM}_{2.5}, \mathrm{CVD}$ mortality increased, ranging from $0.24 \%(95 \%$ CI $0.05,0.43)$ on 40 th day after exposure to $6.11 \%(95 \%$ CI $1.76,10.64)$ per interquartile (IQR) increase $\left(31.5 \mu \mathrm{g} / \mathrm{m}^{3}\right)$ in $\mathrm{PM}_{2.5}$ at moving averages for the previous 3 days $[31,33]$.

The national prospective cohort study conducted in China, which included 189,793 men aged 40 years and above, reported a hazard ratio (HR) for CVD mortality for every $10 \mu \mathrm{g} / \mathrm{m}^{3}$ increase in $\mathrm{PM}_{2.5}$ of $1.09(95 \%$ CI 1.08, 1.10) for a 6-year time period [43]. A time-series study from Guangzhou with a sample size of 33,721 adults reported that the excess risk (ER) of CVD mortality was $6.11 \%$ (95\% CI 1.76, 10.64) per IQR increase in $\mathrm{PM}_{2.5}$ at moving averages for the previous 3 days [31]. A study of 145,477 adults aged 45 years and above conducted in Beijing reported the estimated percentage increase in the risk of death for every $10 \mu \mathrm{g} / \mathrm{m}^{3}$ increase in $\mathrm{PM}_{2.5}$. They found that the risk of CVD mortality rose by $0.24 \%(95 \%$ CI $0.05,0.43)$, cerebrovascular disease (CBD) mortality by $0.23 \%(95 \% \mathrm{CI}(-) 0.03,0.50)$, and ischemic heart disease (IHD) mortality by $0.22 \%(95 \%$ CI $0.06,0.50)$ over $0-5$ days [33]. For every $10 \mu \mathrm{g} / \mathrm{m}^{3}$ increase in $\mathrm{PM}_{2.5}$, a study in Shenyang, China found an increase in CVD mortality of $0.42 \%$ (95\% CI 0.10, 0.73) [35]. In contrast, a study conducted in Chongqing, China found no association between CVD mortality and $\mathrm{PM}_{2.5}$ [37].

The only Mexican study reported that for every $10 \mu \mathrm{g} / \mathrm{m}^{3}$ increase in $\mathrm{PM}_{2.5}$, there was a $3.4 \%(95 \%$ CI $0.67,6.18)$ increase in CVD mortality [27]. The only study from India reported a burden of 5700 $(95 \%$ CI 2800,7500$)$ annual premature deaths $\left(0.16 \%\right.$ of the population) attributable to $\mathrm{PM}_{2.5}$ exposure, of which $29 \%$ and $18 \%$ were IHD and stroke, respectively. The estimated premature deaths per year from ambient $\mathrm{PM}_{2.5}$ exposure in Varanasi, India by IHD was $1600(95 \%$ CI 600, 2200) and stroke was $1000\left(95 \%\right.$ CI 500, 1400) [38]. A Brazilian study reported that every $10 \mu \mathrm{g} / \mathrm{m}^{3}$ increase in $\mathrm{PM}_{2.5}$ resulted in a relative risk (RR) of $1.81 \%(95 \%$ CI $0.03,3.61)$ for CVD mortality [36]. 


\subsection{Hospital Admissions and Emergency Room Visits}

There were four studies [29,32,34,36], one from China and three from Brazil that evaluated hospital admissions and ERV. All reported significant effects of long-term $\mathrm{PM}_{2.5}$ exposure.

In Brazil, the RR was $2.64\left(95 \%\right.$ CI 1.60, 3.69) for hospitalizations related to $\mathrm{PM}_{2.5}$ over a period of 10 days [36]. Another study in Brazil reported a significant increase in the risk of hospitalization for circulatory system diseases of $19.6 \%\left(95 \%\right.$ CI 6.4, 34.6) per $10 \mu \mathrm{g} / \mathrm{m}^{3}$ increase in $\mathrm{PM}_{2.5} 5$ days after exposure [29]. The third study in Brazil similarly reported a 15\% (SE 0.07\%) increase in the risk of hospitalization for CVD per $10 \mu \mathrm{g} / \mathrm{m}^{3}$ increase in $\mathrm{PM}_{2.5}$ concentration 5 days after exposure [34].

The only Chinese study found that an IQR increase in $\mathrm{PM}_{2.5}\left(43.0 \mu \mathrm{g} / \mathrm{m}^{3}\right)$ was associated with $0.3 \%(95 \%$ CI $(-) 2.4,3.0)$ total CVD ERV for 11-day moving average [32].

\subsection{CVD Risk Factors}

Of the five studies that evaluated CVD risk factors [28,39-42], including T2DM, all reported significant effects of long-term $\mathrm{PM}_{2.5}$ exposure. Four of these studies were conducted in China, and one study was conducted in Bulgaria. The following CVD risk factors were assessed by studies included in this review: hypertension $(n=1)$, tachycardia and resting heart rate $(n=1)$, insulin resistance and $\operatorname{T2DM}(n=3)$.

A study conducted in China among adults aged 50 years and above found that for every $10 \mu \mathrm{g} / \mathrm{m}^{3}$ increase in $\mathrm{PM}_{2.5}$ over a 3-year period, the adjusted OR for hypertension was 1.14 (95\% CI 1.07, 1.22). Each $10 \mu \mathrm{g} / \mathrm{m}^{3}$ increase in ambient $\mathrm{PM}_{2.5}$ was associated with a $1.04 \mathrm{mmHg}(95 \% \mathrm{CI} 0.31,1.78)$ increase in diastolic blood pressure and a $1.30 \mathrm{mmHg}(95 \%$ CI 0.04, 3.56) increase in systolic blood pressure [39]. A cross-sectional survey among adults between 20 and 49 years, also from China over a 3-year period, reported, for every $10 \mu \mathrm{g} / \mathrm{m}^{3}$ increase in $\mathrm{PM}_{2.5}$, that the OR for tachycardia was 1.018 (95\% CI 1.017, 1.020) and resting heart rate increased by $0.076 \mathrm{bpm}(95 \% \mathrm{CI} 0.073,0.079)$ [41]. Another Chinese study found that $\mathrm{PM}_{2.5}$ was significantly associated with increased diabetes by OR: $1.14(95 \% \mathrm{CI} 1.03,1.25)$ per IQR increase $\left(26 \mu \mathrm{g} / \mathrm{m}^{3}\right)$ in $\mathrm{PM}_{2.5}$ over a 3-year period [42].

A study in China among adults aged 45 years and above reported that an annual average IQR increase $\left(41.1 \mu \mathrm{g} / \mathrm{m}^{3}\right)$ in $\mathrm{PM}_{2.5}$ was significantly associated with increased T2DM prevalence, $\mathrm{PR}=1.14$ (95\% CI 1.08, 1.20), elevated levels of fasting glucose by $0.26 \mathrm{mmol} / \mathrm{L} \mathrm{(95 \%} \mathrm{CI} \mathrm{0.19,} \mathrm{0.32),} \mathrm{and} \mathrm{elevated}$ $\mathrm{HbA} 1 \mathrm{c}$ by $0.08 \%(95 \%$ CI $0.06,0.10)$ [40]. A study from Bulgaria reported that $\mathrm{PM}_{2.5}$ was positively associated with T2DM, OR 1.32 (95\% CI 0.28, 6.24) [28].

\section{Discussion}

We identified just 17 studies conducted in LMICs with the link between long-term $\mathrm{PM}_{2.5}$ exposure and CMD, and most of the studies (65\% of studies) were in China. This is concerning because LMICs carry the greatest burden of both CMD and air pollution, and most of the studies we reviewed reported significant increases in CVD mortality with increasing $\mathrm{PM}_{2.5}$ levels. The Global Burden of Disease (GBD) in 2015 and Global Health observatory data repository reported that the population-weighted mean exposure to $\mathrm{PM}_{2.5}$ in China, India, Brazil, Mexico, and Bulgaria were $58.4 \mu \mathrm{g} / \mathrm{m}^{3}$ (95\% CI 58.1, 58.7), $74.3 \mu \mathrm{g} / \mathrm{m}^{3}$ (95\% CI 73.9, 74.8), $11.4 \mu \mathrm{g} / \mathrm{m}^{3}$ (95\% CI 11.2, 11.5), $20.1 \mu \mathrm{g} / \mathrm{m}^{3}$ (95\% CI 16.7, 27.2), and $18.8 \mu \mathrm{g} / \mathrm{m}^{3}(95 \%$ CI 18.3, 20.6), respectively. In our review, all the included studies indicated higher levels of $\mathrm{PM}_{2.5}$ than reported by the GBD except for one study from Brazil [29], which reported slightly lower estimates. In this review, $\mathrm{PM}_{2.5}$ estimates reported by Chinese studies ranged from 33.7 to $147 \mu \mathrm{g} / \mathrm{m}^{3}$; Indian $\mathrm{PM}_{2.5}$ estimates ranged from 94.4 to $136.9 \mu \mathrm{g} / \mathrm{m}^{3}$; Brazilian $\mathrm{PM}_{2.5}$ estimates ranged from 4.4 to $23.8 \mu \mathrm{g} / \mathrm{m}^{3}$; Mexican $\mathrm{PM}_{2.5}$ estimates was $27.0 \mu \mathrm{g} / \mathrm{m}^{3}$, and Bulgarian study reported $66.8 \mu \mathrm{g} / \mathrm{m}^{3}$, all of which were much higher than the values reported by the GBD study [4]. Therefore, the GBD estimates may be underestimating CVD deaths attributable to $\mathrm{PM}_{2.5}$ in the countries of focus. Similarly, a global review and meta-regression analysis also observed much lower levels of $\mathrm{PM}_{2.5}$ in China compared to our estimates [45]. Most (70\%) of the research studies included in this review were 
conducted in major cities and industrial centers in China, including Beijing, Chongqing, Guangzhou, Liaoning, Pearl River Delta region, Shanghai, and Shenyang, which may at least partially explain this deviance from the GBD estimates. Satellite-based estimates must be used cautiously and following shortcomings must be kept in mind: underestimation of ground measurements in locations with higher concentrations like East Asia, South Asia, North Africa, and Sub-Saharan Africa [46].

The duration of exposure ascertainment to outcome varied from a 3-day average to a 13-year average. The effect estimates varied with duration of exposure- longer exposure periods associated with smaller effect estimates. We observed differences among the effect estimates for cardiovascular mortality for annual averages or longer duration and less than annual averages. In our review, we observed that the effect estimates were smaller when the exposure window was longer, which needs further investigation. This was similar to a global meta-analysis of cohort studies, which also found that the effect estimates decreased with increasing concentrations [45].

This review was focused on CMDs as we currently lack literature on the effects of long-term exposure to $\mathrm{PM}_{2.5}$ on CMDs in LMIC settings. Globally, $17.1 \%$ of IHD mortality and $14.2 \%$ of CBD mortality in 2015 was attributable to $\mathrm{PM}_{2.5}$ as reported by GBD [7]. A prospective European cohort study found an increased risk of stroke associated with a $5 \mu \mathrm{g} / \mathrm{m}^{3}$ increase in $\mathrm{PM}_{2.5}$ with a hazard ratio of $1.19(95 \%$ CI $0.88,1.62)$ [13]. In our review, Luo et al. from their Beijing study reported that every $10 \mu \mathrm{g} / \mathrm{m}^{3}$ increase in $\mathrm{PM}_{2.5}$ was associated with an increased risk of CBD mortality of $0.23 \%(95 \% \mathrm{CI}$ $(-) 0.03,0.50)[33]$.

Across all of the included studies, the results ranged from $0.24 \%$ [33] to $6.11 \%$ [31] increase in CVD mortality for every $10 \mu \mathrm{g} / \mathrm{m}^{3}$ increase in $\mathrm{PM}_{2.5}$. While this effect estimate is relatively small, the ubiquitous nature of the exposure is likely to result in a large population-attributable disease burden. This range of values was higher than a recent meta-regression analysis of 53 studies, that found the percent increase at mean exposure of $10 \mu \mathrm{g} / \mathrm{m}^{3} \mathrm{PM}_{2.5}$ associated with a $1.46 \%(95 \% \mathrm{CI}$ $1.25,1.67)$ increase in CVD mortality [45]. Similarly, a review of European epidemiological studies reported that the relative risk of CVD mortality was 1.11 (95\% CI 1.07, 1.15) for a quartile increase in $\mathrm{PM}_{2.5}$ [47]. Another global review also noted that cardiorespiratory mortality was increased by $2.29 \%$ $(95 \%$ CI $1.36,3.85)$ per $10 \mu \mathrm{g} / \mathrm{m}^{3}$ increase in $\mathrm{PM}_{2.5}$ [48]. The effect estimates depended on the type of exposure assessment method as using ground-level monitors produced estimates $44-46 \%$ lower than that of hybrid space-time model [45]. Most of the studies reviewed here utilized secondary data from stationary monitoring stations (seven studies) or were modeled estimates (eight studies), which might not accurately reflect personal exposures. The only Indian study reported that premature mortality per year in the city of Varanasi from IHD was 1600 (95\% CI 600, 2200) and stroke was 1000 (95\% CI 500, 1400) after exposure to $\mathrm{PM}_{2.5}$ that could be saved [38]. There were no Indian studies measuring the impact of ambient air pollution on cardio-metabolic health outcomes, which needs focus in the future studies, given the fact that air pollution levels are alarmingly high, especially in the Indo-Gangetic plain [49].

A strong association between $\mathrm{PM}_{2.5}$ and CVD hospital admissions was observed. A pooled estimate from European countries reported that relative risk of cardiovascular hospitalizations increased by $1.8 \%(95 \%$ CI $0.1,3.4)$ per IQR increase in $\mathrm{PM}_{2.5}\left(12.4 \mu \mathrm{g} / \mathrm{m}^{3}\right)$ [50]. Results from a global review and meta-analysis showed that hospital admissions due to cardiorespiratory diseases were increased by $1.64 \%\left(95 \%\right.$ CI 1.06, 2.53) per $10 \mu \mathrm{g} / \mathrm{m}^{3}$ increase in $\mathrm{PM}_{2.5}$ [48], which was much lower than the studies from Brazil identified in this review that reported a 15-19.6\% increase in CVD hospitalizations [29,34]. On the contrary, a Chinese study observed that total and severe CVD ERVs increased by $0.3 \%$ (95\% CI $(-) 2.4,3.0)$ and decreased by $0.1 \%\left(95 \%\right.$ CI 3.4, 3.3) for an IQR increase in $\mathrm{PM}_{2.5}$ by $43.0 \mu \mathrm{g} / \mathrm{m}^{3}$ in 11-day moving average, respectively, which indicated delayed association [32]. The varying effect estimates could be attributed to the population included and the exposure assessment method used in these studies.

Long-term exposure showed the strongest associations with hypertension. In a meta-analysis, there was a statistically insignificant increase in hypertension risk $(\mathrm{OR}=1.065,95 \% \mathrm{CI} 0.985-1.152)$ 
with each $10 \mu \mathrm{g} / \mathrm{m}^{3}$ increment in $\mathrm{PM}_{2.5}$ [17]. We found very similar results from the Chinese study by Lin et al. who reported an increased risk of hypertension by an OR $=1.14(95 \%$ CI 1.07, 1.22) with each $10 \mu \mathrm{g} / \mathrm{m}^{3}$ increment in $\mathrm{PM}_{2.5}$ [39]. A meta-analysis among cohort studies reported a positive association for T2DM and relative risk of 1.25 (95\% CI 1.10, 1.43) for every $10 \mu \mathrm{g} / \mathrm{m}^{3}$ increase in $\mathrm{PM}_{2.5}$. Another review of European and North American studies reported that the pooled relative risk estimate for T2DM per $10 \mu \mathrm{g} / \mathrm{m}^{3}$ increase in $\mathrm{PM}_{2.5}$ was $1.10(95 \%$ CI 1.02, 1.18) [15]. The Chinese study from our review reported that for an IQR increase in $\mathrm{PM}_{2.5}$, T2DM prevalence increased by $\mathrm{PR}=1.14(95 \%$ CI $1.08,1.20)$ [40]. There were no studies conducted on lipid disorders with respect to air pollution exposure.

In this review, about two-thirds of the studies included were conducted in the last three years (2016-2018) indicating that this area is gaining more focus recently. A prospective cohort study design is considered the gold standard, but only one study, which was conducted in China, used a cohort design [43]. Further studies examining personal exposure to $\mathrm{PM}_{2.5}$ or statistically modeled estimates for $\mathrm{PM}_{2.5}$ and CMDs in LMICs are in urgent need to help develop scientific literature.

This study is not without its limitations, firstly the heterogeneity of identified studies prohibited the use of meta-analytical methods to produce summary estimates of effect. We focused on long-term exposures considering that in LMICs, ambient air pollution is chronic exposure and effects may be larger for long-term exposures versus short-term exposures. From our review results, we cannot conclude this assumption, as we found that for a longer duration of exposure, the effect estimates were smaller, and further investigation is needed to confirm this. The second limitation is that we focused solely on one component of ambient air pollution, $\mathrm{PM}_{2.5}$, yet individuals are exposed to complex mixtures. We chose to focus on $\mathrm{PM}_{2.5}$ versus $\mathrm{PM}_{10}$ because previous research has demonstrated a stronger effect on CVD due to the ability of $\mathrm{PM}_{2.5}$ particles to travel to distant organs [14]. Moreover, most included studies used a single-pollutant model, but a few also measured other pollutants, including $\mathrm{PM}_{10}, \mathrm{PM}_{1}$, sulfur dioxide $\left(\mathrm{SO}_{2}\right)$, nitrogen dioxide $\left(\mathrm{NO}_{2}\right)$, nitrous oxide $(\mathrm{NO})$, carbon monoxide $(\mathrm{CO})$, ozone $\left(\mathrm{O}_{3}\right)$, black carbon, organic and elemental carbon, soluble ions, and noise and traffic pollution. We evaluated only the effect estimates of $\mathrm{PM}_{2.5}$ here. The third limitation is the type of studies included in this review is very different and, hence, the effect sizes should be interpreted with caution. Future research should expand to include multipollutant models that take into account potential interactions between pollutants, as well as source-specific effects.

In conclusion, few studies have evaluated the association between long-term exposure to $\mathrm{PM}_{2.5}$ and CMD outcomes in LMICs, and the majority of this literature has come from China. We did not identify a single study conducted in North and Sub-Saharan Africa, which is home to $17 \%$ of the world's population. Considering that the vast majority of morbidity and mortality attributable to $\mathrm{PM}_{2.5}$ is in LMICs, this represents a major environmental injustice. The global environmental health community must drive a strong research agenda relating to the CMD effects of $\mathrm{PM}_{2.5}$ in these settings in order to provide context-specific evidence to policymakers in these countries.

Author Contributions: The article was conceptualized by S.J. and D.P.; designing, methodology, data extraction and preparing tables and figure was done by S.J., L.M.J., M.M., G.K.W., N.L.S. and S.S.H.; S.J., L.M.J and N.L.S. wrote the manuscript R.S., P.K.D., J.S., D.P., reviewed the manuscript and; the effort was supervised by D.P.

Funding: This research was funded by NIH Fogarty International Center, U2R TW010108 and U01 TW010097 and The APC was funded by GEOHealth grant no: U01 TW010097.

Acknowledgments: The work reported in this publication was supported by the NIH Fogarty International Center under Award Numbers U2R TW010108 and U01 TW010097.

Conflicts of Interest: The authors declare no conflict of interest. 


\section{Abbreviations}

CBD-Cerebrovascular Disease;

CI-Confidence Interval;

CMD-Cardio-Metabolic Diseases;

CO-Carbon Monoxide;

CVD-Cardiovascular Diseases;

DBP-Diastolic Blood Pressure;

DM/T2DM-Diabetes Mellitus/Type 2 Diabetes Mellitus;

ER-Excess Risk; ERV-Emergency Room Visits;

HbA1c-HemoglobinA1c;

HIC-High Income Countries;

HR-Hazard Ratio;

IHD-Ischemic Heart Disease;

IQR-Interquartile Range;

LMIC-Low-and Middle-Income Countries;

$\mathrm{NO}_{2}$-Nitrogen Dioxide;

NO-Nitrous Oxide;

$\mathrm{O}_{3}$-Ozone; OR-Odds Ratio;

PM-Particulate Matter;

RR-Relative Risk;

SBP-Systolic Blood Pressure;

SD-Standard Deviation;

$\mathrm{SO}_{2}$-Sulfur Dioxide;

WHO-World Health Organization.

\section{Appendix A.}

\section{Appendix A.1. Search Terms}

Air pollution: "air pollution" or "particulate matter" or "air pollutants" or "ambient air pollution" or "Total suspended particles" or "particulate matter" or "soot"

CMD: "diabetes" or "diabet*" or "IDDM" or "NIDDM" or "Cardiovascular*" or "Cardiovascular Diseases" or "Cardiovascular Abnormalities" or "Heart Arrest" or "Cardiac arrest" or "Arterial Occlusive Diseases" or "Cerebrovascular Disorders" or "Hypertens*" or "Myocardial Ischemia" or "Prehypertens*" or "Angina*" or "Glucose Metabolism Disorders" or "Diabetes*" or "Prediabet" or "Hyperglycem*" or "Glucose Intoleran*" or "Insulin Resistan*" or "Lipoprotein*" or "Apolipoprotein*" or "Chylomicrons" or "Chylomicron Remnants" or "Cholesterol*" or "High-Density Lipoproteins, Pre-beta" or "Cardiac Death" or "Asphyxia" or "Brain Death" or "Death, Sudden" or "Death, Sudden, Cardiac" or "Sudden Cardiac Death" or "Sudden Cardiac Arrest" or "Cardiac Arrest, Sudden"

Countries: Low- and Middle- Income Countries (Low income, lower middle income, upper middle-income countries) and "NOT" High Income Countries (see Table A1 for detailed search strategy). 
Appendix A.2. Cochrane Risk of Bias Tool

Table A1. Low risk of bias.

\begin{tabular}{|c|c|c|c|c|c|}
\hline & Study Title & Selection & Exposure Assessment & Confounders & Outcome Assessment \\
\hline 1 & Borja-Aburto 1998 & & & & \\
\hline 2 & Dzhambov 2016 & & & & \\
\hline 3 & Ferreira 2016 & & & & \\
\hline 4 & Jain 2017 & & & & \\
\hline 5 & Lin 2016 & & & & \\
\hline 6 & Lin 2016 & & & & \\
\hline 7 & Lin 2017 & & & & \\
\hline 8 & Liu 2013 & & & & \\
\hline 9 & Liu 2016 & & & & \\
\hline 10 & Luo 2016 & & & & \\
\hline 11 & Mantovani 2016 & & & & \\
\hline 12 & Meng 2013 & & & & \\
\hline 13 & Rodrigues 2017 & & & & \\
\hline 14 & Venners 2003 & & & & \\
\hline 15 & Xie 2018 & & & & \\
\hline 16 & Yang 2018 & & & & \\
\hline 17 & Yin 2017 & & & & \\
\hline
\end{tabular}

\section{References}

1. Collaborators GCoD. Global, regional, and national age-sex specific mortality for 264 causes of death, 1980-2016: A systematic analysis for the Global Burden of Disease Study 2016. Lancet 2017, 390, 1151-1210. [CrossRef]

2. Brook, R.D.; Rajagopalan, S.; Pope, C.A., 3rd; Brook, J.R.; Bhatnagar, A.; Diez-Roux, A.V.; Holguin, F.; Hong, Y.; Luepker, R.V.; Mittleman, A.M.; et al. Particulate matter air pollution and cardiovascular disease: An update to the scientific statement from the American Heart Association. Circulation 2010, 121, 2331-2378. [CrossRef] [PubMed]

3. Dockery, D.W.; Stone, P.H. Cardiovascular risks from fine particulate. Air Pollut. 2007, 356, 511-513.

4. Cohen, A.J.; Brauer, M.; Burnett, R.; Anderson, H.R.; Frostad, J.; Estep, K.; Balakrishnan, K.; Brunekreef, B.; Dandona, L.; Dandona, R.; et al. Estimates and 25-year trends of the global burden of disease attributable to ambient air pollution: An analysis of data from the Global Burden of Diseases Study 2015. Lancet 2017, 389, 1907-1918. [CrossRef]

5. Shah, A.S.; Langrish, J.P.; Nair, H.; McAllister, D.A.; Hunter, A.L.; Donaldson, K.; Newby, D.E.; Mills, N.L. Global association of air pollution and heart failure: A systematic review and meta-analysis. Lancet 2013, 382, 1039-1048. [CrossRef]

6. Shah, A.S.; Lee, K.K.; McAllister, D.A.; Hunter, A.; Nair, H.; Whiteley, W.; Langrish, J.P.; Newby, D.E.; Mills, N.L. Short term exposure to air pollution and stroke: Systematic review and meta-analysis. BMJ 2015, 350, h1295. [CrossRef] [PubMed]

7. Forouzanfar, M.H.; Afshin, A.; Alexander, T.L.; Anderson, H.R.; Bhutta, Z.A.; Biryukov, S.; Brauer, R.; Cercy, K.; Charlson, F.J.; Cohen, A.J.; et al. Global, regional, and national comparative risk assessment of 79 behavioural, environmental and occupational, and metabolic risks or clusters of risks, 1990-2015: A systematic analysis for the Global Burden of Disease Study 2015. Lancet 2016, 388, 1659-1724. [CrossRef]

8. Mustafic, H.; Jabre, P.; Caussin, C.; Murad, M.H.; Escolano, S.; Tafflet, M.; Perier, M.-C.; Marijon, E.; Vernerey, D.; Empana, J.-P.; et al. Main air pollutants and myocardial infarction: A systematic review and meta-analysis. JAMA 2012, 307, 713-721. [CrossRef] [PubMed]

9. Dockery, D.W.; Pope, C.A., 3rd; Xu, X.; Spengler, J.D.; Ware, J.H.; Fay, M.E.; Ferris, B.G.; Speizer, F.E. An association between air pollution and mortality in six U.S. cities. N. Engl. J. Med. 1993, 329, 1753-1759. [CrossRef]

10. World Health Orgnaisation (WHO). Ambient Air Pollution: A Global Assessment of Exposure and Burden of Disease. 2016. Available online: https://apps.who.int/iris/bitstream/handle/10665/250141/9789241511353eng.pdf?sequence=1 (accessed on 15 February 2019). 
11. Pope, C.A., 3rd; Dockery, D.W. Health effects of fine particulate air pollution: Lines that connect. J. Air Waste Manag. Assoc. 2006, 56, 709-742. [CrossRef]

12. Pope, C.A., 3rd; Thun, M.J.; Namboodiri, M.M.; Dockery, D.W.; Evans, J.S.; Speizer, F.E.; Heath, C.W. Particulate air pollution as a predictor of mortality in a prospective study of U.S. adults. Am. J. Resp. Crit. Care Med. 1995, 151, 669-674. [CrossRef] [PubMed]

13. Stafoggia, M.; Cesaroni, G.; Peters, A.; Andersen, Z.J.; Badaloni, C.; Beelen, R.; Caracciolo, B.; Cyrys, J.; de Faire, U.; de Hoogh, K.; et al. Long-term exposure to ambient air pollution and incidence of cerebrovascular events: Results from 11 European cohorts within the ESCAPE project. Environ. Health Perspect. 2014, 122, 919-925. [CrossRef] [PubMed]

14. Hoek, G.; Krishnan, R.M.; Beelen, R.; Peters, A.; Ostro, B.; Brunekreef, B.; Brunekreef, B.; Kaufman, D.J. Long-term air pollution exposure and cardio-respiratory mortality: A review. Environ. Health 2013, 12, 43. [CrossRef] [PubMed]

15. Eze, I.C.; Hemkens, L.G.; Bucher, H.C.; Hoffmann, B.; Schindler, C.; Kunzli, N.; Schikowski, T.; Probst-Hensch, N.M. Association between ambient air pollution and diabetes mellitus in Europe and North America: Systematic review and meta-analysis. Environ. Health Perspect. 2015, 123, 381-389. [CrossRef] [PubMed]

16. Balti, E.V.; Echouffo-Tcheugui, J.B.; Yako, Y.Y.; Kengne, A.P. Air pollution and risk of type 2 diabetes mellitus: A systematic review and meta-analysis. Diabetes Res. Clin. Pract. 2014, 106, 161-172. [CrossRef] [PubMed]

17. Cai, Y.; Zhang, B.; Ke, W.; Feng, B.; Lin, H.; Xiao, J.; Zeng, W.; Li, X.; Tao, J.; Yung, Z.; et al. Associations of short-term and long-term exposure to ambient air pollutants with hypertension: A systematic review and meta-analysis. Hypertension 2016, 68, 62-70. [CrossRef]

18. Yamamoto, S.S.; Phalkey, R.; Malik, A.A. A systematic review of air pollution as a risk factor for cardiovascular disease in South Asia: Limited evidence from India and Pakistan. Int. J. Hygiene Environ. Health 2014, 217, 133-144. [CrossRef]

19. MHFW (Ministry of Health and Family Welfare). New Delhi, India: Ministry of Health and Family Welfare, Government of India (August 2015); 2015. Report of the Steering Committee on Air Pollution and Health-Related Issues. Available online: http://www.mohfw.nic.in/showfile.php?lid=3650 (accessed on 17 December 2018).

20. Health Effects Institute. State of Global Air 2018; Special Report; Health Effects Institute: Boston, MA, USA, 2018.

21. Pescatello, L.S.; VanHeest, J.L. Physical activity mediates a healthier body weight in the presence of obesity. Br. J. Sports Med. 2000, 34, 86-93. [CrossRef]

22. Nair, M.; Ali, M.K.; Ajay, V.S.; Shivashankar, R.; Mohan, V.; Pradeepa, R.; Deepa, M.; Khan, M.M.; Fatmi, Z.A.; Reddy, K.S.; et al. CARRS Surveillance study: Design and methods to assess burdens from multiple perspectives. BMC Public Health 2012, 12, 701. [CrossRef]

23. World Bank Country and Lending Groups. Available online: https://datahelpdesk.worldbank.org/ knowledgebase/articles/906519 (accessed on 1 February 2018).

24. Counsell, C. Formulating questions and locating primary studies for inclusion in systematic reviews. Ann. Intern. Med. 1997, 127, 380-387. [CrossRef]

25. Sterne, J.A.; Hernan, M.A.; Reeves, B.C.; Savovic, J.; Berkman, N.D.; Viswanathan, M.; Henry, D.; Altman, D.G.; Ansari, M.T.; Boutron, I.; et al. ROBINS-I: A tool for assessing risk of bias in non-randomised studies of interventions. BMJ 2016, 355, i4919. [CrossRef] [PubMed]

26. Moher, D.; Shamseer, L.; Clarke, M.; Ghersi, D.; Liberati, A.; Petticrew, M.; Shekelle, P.; Stewart, L.A.; PRISMA-P Group. Preferred reporting items for systematic review and meta-analysis protocols (PRISMA-P) 2015 statement. Syst Rev. 2015, 4, 1. [CrossRef] [PubMed]

27. Borja-Aburto, V.H.; Castillejos, M.; Gold, D.R.; Bierzwinski, S.; Loomis, D. Mortality and ambient fine particles in southwest Mexico City, 1993-1995. Environ. Health Perspect. 1998, 106, 849-855. [CrossRef] [PubMed]

28. Ferreira, T.M.; Forti, M.C.; de Freitas, C.U.; Nascimento, F.P.; Junger, W.L.; Gouveia, N. Effects of particulate matter and its chemical constituents on elderly hospital admissions due to circulatory and respiratory diseases. Int. J. Environ. Res. Public Health 2016, 13, 947. [CrossRef] [PubMed] 
29. Lin, H.; Liu, T.; Xiao, J.; Zeng, W.; Li, X.; Guo, L.; Zhang, Y.; Xu, Y.; Tao, J.; Xian, H.; et al. Mortality burden of ambient fine particulate air pollution in six Chinese cities: Results from the Pearl River Delta study. Environ. Int. 2016, 96, 91-97. [CrossRef] [PubMed]

30. Lin, H.; Tao, J.; Du, Y.; Liu, T.; Qian, Z.; Tian, L.; Di, Q.; Rutherford, S.; Guo, L.; Zeng, W.; et al. Particle size and chemical constituents of ambient particulate pollution associated with cardiovascular mortality in Guangzhou, China. Environ. Pollut. 2016, 208 Pt B, 758-766. [CrossRef]

31. Liu, L.; Breitner, S.; Schneider, A.; Cyrys, J.; Bruske, I.; Franck, U.; Schlink, U.; Leitte, A.M.; Herbarth, O.; Wiedensohler, A.; et al. Size-fractioned particulate air pollution and cardiovascular emergency room visits in Beijing, China. Environ. Res. 2013, 121, 52-63. [CrossRef] [PubMed]

32. Luo, K.; Li, W.; Zhang, R.; Li, R.; Xu, Q.; Cao, Y. Ambient fine particulate matter exposure and risk of cardiovascular mortality: Adjustment of the meteorological factors. Int. J. Environ. Res. Public Health 2016, 13, 1082. [CrossRef]

33. Mantovani, K.C.; Nascimento, L.F.; Moreira, D.S.; Vieira, L.C.; Vargas, N.P. Air pollutants and hospital admissions due to cardiovascular diseases in Sao Jose do Rio Preto, Brazil. Ciencia Saude Coletiva 2016, 21, 509-515. [CrossRef]

34. Meng, X.; Ma, Y.; Chen, R.; Zhou, Z.; Chen, B.; Kan, H. Size-fractionated particle number concentrations and daily mortality in a Chinese city. Environ. Health Perspect. 2013, 121, 1174-1178. [CrossRef]

35. Rodrigues, P.C.O.; Pinheiro, S.L.; Junger, W.; Ignotti, E.; Hacon, S.S. Climatic variability and morbidity and mortality associated with particulate matter. Revista de Saude Publica 2017, 51, 91. [CrossRef] [PubMed]

36. Venners, S.A.; Wang, B.; Xu, Z.; Schlatter, Y.; Wang, L.; Xu, X. Particulate matter, sulfur dioxide, and daily mortality in Chongqing, China. Environ. Health Perspect. 2003, 111, 562-567. [CrossRef] [PubMed]

37. Jain, V.; Dey, S.; Chowdhury, S. Ambient PM2.5 exposure and premature mortality burden in the holy city Varanasi, India. Environ. Pollut. 2017, 226, 182-189. [CrossRef] [PubMed]

38. Lin, H.; Guo, Y.; Zheng, Y.; Di, Q.; Liu, T.; Xiao, J.; Li, X.; Zeng, W.; Cummings-Vaughn, L.A.; Howard, S.W.; et al. Long-term effects of ambient pm2.5 on hypertension and blood pressure and attributable risk among older Chinese Adults. Hypertension 2017, 69, 806-812. [CrossRef] [PubMed]

39. Liu, C.; Yang, C.; Zhao, Y.; Ma, Z.; Bi, J.; Liu, Y.; Meng, X.; Wang, Y.; Cai, J.; Kan, H.; et al. Associations between long-term exposure to ambient particulate air pollution and type 2 diabetes prevalence, blood glucose and glycosylated hemoglobin levels in China. Environ. Int. 2016, 92, 416-421. [CrossRef] [PubMed]

40. Xie, X.; Wang, Y.; Yang, Y.; Xu, J.; Zhang, Y.; Tang, W.; Guo, T.; Wang, Q.; Shen, H.; Zhang, Y.; et al. Long-term exposure to fine particulate matter and tachycardia and heart rate: Results from 10 million reproductive-age adults in China. Environ. Pollut. 2018, 242 Pt B, 1371-1378. [CrossRef]

41. Yang, B.Y.; Qian, Z.M.; Li, S.; Chen, G.; Bloom, M.S.; Elliott, M.; Syberg, K.W.; Heinrich, J.; Markevych, I.; Wang, S.-Q.; et al. Ambient air pollution in relation to diabetes and glucose-homoeostasis markers in China: A cross-sectional study with findings from the 33 Communities Chinese Health Study. Lancet Planet. Health 2018, 2, e64-e73. [CrossRef]

42. Yin, P.; Brauer, M.; Cohen, A.; Burnett, R.T.; Liu, J.; Liu, Y.; Liang, R.; Wang, W.; Qi, J.; Wang, L.; et al. Long-term fine particulate matter exposure and nonaccidental and cause-specific mortality in a large national Cohort of Chinese Men. Environ. Health Perspect. 2017, 125, 117002. [CrossRef] [PubMed]

43. Xie, W.; Li, G.; Zhao, D.; Xie, X.; Wei, Z.; Wang, W.; Wang, M.; Li, G.; Liu, W.; Sun, J.; et al. Relationship between fine particulate air pollution and ischaemic heart disease morbidity and mortality. Heart 2015, 101, 257-263. [CrossRef] [PubMed]

44. Vodonos, A.; Awad, Y.A.; Schwartz, J. The concentration-response between long-term PM2. 5 exposure and mortality; A meta-regression approach. Environ. Res. 2018, 166, 677-689. [CrossRef] [PubMed]

45. World Bank and Institute for Health Metrics and Evaluation. The Cost of Air Pollution: Strengthening the Economic Case for Action; License: Creative Commons Attribution CC BY 3.0 IGO; World Bank: Washington, DC, USA, 2016.

46. Pelucchi, C.; Negri, E.; Gallus, S.; Boffetta, P.; Tramacere, I.; La Vecchia, C. Long-term particulate matter exposure and mortality: A review of European epidemiological studies. BMC Public Health 2009, 9, 453. [CrossRef] [PubMed]

47. Requia, W.J.; Adams, M.D.; Arain, A.; Papatheodorou, S.; Koutrakis, P.; Mahmoud, M. Global association of air pollution and cardiorespiratory diseases: A systematic review, meta-analysis, and investigation of modifier variables. Am. J. Public Health 2018, 108, S123-S130. [CrossRef] [PubMed] 
48. Balakrishnan, K.; Dey, S.; Gupta, T.; Dhaliwal, R.S.; Brauer, M.; Cohen, A.J.; Stanaway, J.D.; Beig, G.; Joshi, T.K.; Aggarwal, A.N.; et al. The impact of air pollution on deaths, disease burden, and life expectancy across the states of India: The Global Burden of Disease Study 2017. Lancet Planet. Health 2019, 3, e26-e39. [CrossRef]

49. Lanzinger, S.; Schneider, A.; Breitner, S.; Stafoggia, M.; Erzen, I.; Dostal, M.; Pastorkova, A.; Bastian, S.; Cyrys, J.; Zscheppang, A.; et al. Associations between ultrafine and fine particles and mortality in five central European cities-Results from the UFIREG study. Environ. Int. 2016, 88, 44-52. [CrossRef] [PubMed]

50. He, D.; Wu, S.; Zhao, H.; Qiu, H.; Fu, Y.; Li, X.; He, Y. Association between particulate matter 2.5 and diabetes mellitus: A meta-analysis of cohort studies. J. Diabetes Investig. 2017, 8, 687-696. [CrossRef]

(C) 2019 by the authors. Licensee MDPI, Basel, Switzerland. This article is an open access article distributed under the terms and conditions of the Creative Commons Attribution (CC BY) license (http://creativecommons.org/licenses/by/4.0/). 